\title{
Editorial comment: Prognostic value of diffusion-weighted imaging in patients with newly diagnosed sporadic Creutzfeldt-Jakob disease
}

\author{
Jinhee Jang ${ }^{1}$ (1) \\ Received: 1 November 2021 / Revised: 1 November 2021 / Accepted: 18 November 2021 /Published online: 15 January 2022 \\ (c) The Author(s), under exclusive licence to European Society of Radiology 2022
}

Creutzfeldt-Jakob disease (CJD) is a rare, but fatal neurodegenerative disease, which is caused by accumulation of misfolded prion protein. It affects about $1-2$ persons per year in every million people worldwide [1]. Sporadic CJD (sCJD) is the most common type of CJD [2], and it presents with notoriously rapid progression and about two-thirds of patients die within a year of diagnosis. Moreover, currently, there is no effective treatment for sCJD. Its cruelly rapid progression and the absence of established treatment require an immediate diagnosis. Like other neurodegenerative diseases, a definite diagnosis of SCJD can be made by histological evaluation of brain tissue. However, considering the procedural risk to patients and the potential risk of exposure of the highly infectious tissue to medical staff, a non-invasive diagnostic workup without biopsy is recommended and preferred. To establish diagnostic criteria for probable sCJD, several clinical manifestations and results from diagnostic methods are required. Among them, MRI findings, especially diffusion-weighted image (DWI), are a clinically useful and accurate tool for the diagnosis of SCJD, and for differentiating other types of CJD [3, 4].

While brain MRI could be useful for diagnosing sCJD, image findings as well as clinical features and disease severity could be variable [4-6]. There were several reports demonstrating this relationship between MRI features and disease severity $[4,5]$, suggesting a possible association between DWI findings, disease severity, and clinical progress. In addition, there is still a clinically unmet need for the utilization of those information in clinical practice. In a recent remarkable work by Park et al. [7], they suggested that DWI findings have prognostic value for SCJD with a simple, easy-to-use scoring system to stratify sCJD patients

Jinhee Jang

znee@ catholic.ac.kr

1 Department of Radiology, Seoul St. Mary's Hospital, College of Medicine, The Catholic University of Korea, 222 Banpo-daero, Seocho-gu 06591, Seoul, Korea according to their survival. They revealed that subjects' age ( $\geq 60$-year-old) and caudate nucleus or putamen involvement on DWI were associated with poor prognosis. In addition, they built a scoring system addressing the two key factors (patients' age and the extent of the DWI lesions). Specifically, they found a subgroup with a very short median survival of about 1.7 months; patients in this subgroup were old and had extensive DWI lesions, such as moderate to extensive (3 or more lobes) cortical and striatal involvement.

Of note, as compared to striatal involvement, the severity of cortical involvement was not significantly associated with the prognosis of sCJD. While cortical and basal ganglia involvement were described as common findings of SCJD $[4,6]$, we might speculate that the involvement of basal ganglia on DWI might be followed by cortical involvement. In a study by Park et al. [7], this type of progression was observed in about half of the patients with isolated cortical involvement $(5 / 11,45.5 \%)$. Another study by Eisenmenger et al. [5] showed the greatest signal changes on follow-up DWI in caudate nuclei followed by putamen in sCJD. Another report from Hutzelmen [8] showed progressive lesions in the striatum in T2-weighted images. Also, a recent meta-analysis [4] suggested the involvement of striatum without neocortical involvement is relatively rare (7\%), as compared to isolated cortical involvement $(30 \%)$ or simultaneous cortical and striatal involvement (60\%). Striatal involvement might be a finding in an advanced stage of sCJD, as compared to cortical involvement.

There were reports about the prognostic value of other biomarkers, those were used for the diagnosis of SCJD [9, 10]. A study with relatively large sCJD cases revealed prognostic value in some diagnostic markers such as 14-3-3 in the cerebrospinal fluid [9]. A recent report showed the diagnostic value of novel biomarkers in SCJD such as real-time quaking-induced conversion [11]. Future works combining clinical and radiological biomarkers could be built for the better stratification and prognostication of SCJD patients. In addition, further validation of a scoring system with a large 
population and multi-center design would be required for the usage of this prognostic model.

To conclude, DWI of the brain has both diagnostic and prognostic value for the sCDJ patients. A combination of patients' age and extensive DWI lesions in the striatum and neocortex could suggest an extremely poor prognosis of sCJD patients. An elegant scoring system might be a readily available tool for the sCJD patients. Further validation of these approaches, as well as the development of a comprehensive approach that encompasses clinical and radiological features, is needed.

\section{Funding None.}

\section{Declarations}

Guarantor The scientific guarantor of this publication is Jinhee Jang.

Conflict of interest The authors of this manuscript declare no relationships with any companies, whose products or services may be related to the subject matter of the article.

Statistics and biometry No complex statistical methods were necessary for this paper.

Informed consent Written informed consent was not required for this study because this is an editorial without any study subjects.

Ethical approval Institutional Review Board approval was not required because this is an editorial without any study subjects.

\section{Methodology}

- Editorial comment.

\section{References}

1. Uttley L, Carroll C, Wong R, Hilton DA, Stevenson M (2020) Creutzfeldt-Jakob disease: a systematic review of global incidence, prevalence, infectivity, and incubation. Lancet Infect Dis 20:e2-e10

2. Abrahantes JC, Aerts M, van Everbroeck B et al (2007) Classification of sporadic Creutzfeldt-Jakob disease based on clinical and neuropathological characteristics. Eur J Epidemiol 22:457-465

3. Tschampa HJ, Zerr I, Urbach H (2007) Radiological assessment of Creutzfeldt-Jakob disease. Eur Radiol 17:1200-1211

4. Park HY, Kim M, Suh CH, Kim SY, Shim WH, Kim SJ (2021) Diagnostic value of diffusion-weighted brain magnetic resonance imaging in patients with sporadic Creutzfeldt-Jakob disease: a systematic review and meta-analysis. Eur Radiol 31:9073-9085

5. Eisenmenger L, Porter M-C, Carswell CJ et al (2016) Evolution of diffusion-weighted magnetic resonance imaging signal abnormality in sporadic Creutzfeldt-Jakob disease, with histopathological correlation. JAMA Neurol 73:76-84

6. Fragoso DC, Gonçalves Filho AL, Pacheco FT et al (2017) Imaging of Creutzfeldt-Jakob disease: imaging patterns and their differential diagnosis. Radiographics 37:234-257

7. Park HY, Suh CH, Shim WH et al (2021) Prognostic value of diffusion-weighted imaging in patients with newly diagnosed sporadic Creutzfeldt-Jakob disease. Eur Radiol. https://doi.org/ 10.1007/s00330-021-08363-1

8. Hutzelmann A, Biederer J (1998) MRI follow-up in a case of clinically diagnosed Creutzfeld-Jakob disease. Eur Radiol 8:421-423

9. Llorens F, Rübsamen N, Hermann P et al (2020) A prognostic model for overall survival in sporadic Creutzfeldt-Jakob disease. Alzheimers Dement 16:1438-1447

10. Pocchiari M, Puopolo M, Croes EA et al (2004) Predictors of survival in sporadic Creutzfeldt-Jakob disease and other human transmissible spongiform encephalopathies. Brain 127:2348-2359

11. Bizzi A, Pascuzzo R, Blevins J et al (2020) Evaluation of a new criterion for detecting prion disease with diffusion magnetic resonance imaging. JAMA Neurol 77:1141-1149

Publisher's Note Springer Nature remains neutral with regard to jurisdictional claims in published maps and institutional affiliations. 